\title{
ANÁLISE DO ALÍVIO TÉRMICO DE TENSÕES RESIDUAIS DO AÇO SAE 4140 POR DIFRAÇÃO DE RAIOS-X FAST IN-SITU*
}

\author{
Rayane Sonda Cassel Bielefeldt ${ }^{1}$ \\ Igor Luis Dieh² \\ Thomas Gabriel Rosauro Clarke ${ }^{3}$
}

\section{Resumo}

As $T^{R} C$ (tensões residuais compressivas) induzidas por shot-peening $(S P)$ podem ser relaxadas e redistribuídas sob cargas cíclicas mecânicas, térmicas e termomecânicas. $O$ objetivo desta pesquisa é avaliar a redução da $T^{R} C$ por $S P$ através da difração de raios- $X$ fast in-situ $\left(D^{\left.R X_{i n-s i t u}\right)}\right.$ na superfície do aço SAE 4140 durante tempo $(t)$ de patamar de $t$ $[s]=54000$ em três diferentes temperaturas $(T)$ de $T[K]=423,16 ; 523,16$ e 623,16 . É investigada a relaxação de $T^{R} C$ por cargas térmicas, mantendo as amostras, previamente tratadas por $S P$, em um forno com diferentes temperaturas de patamar, enquanto são medidos os valores de $T^{R}$ ao longo do $t$. Foi construída uma câmara de vácuo acoplada a um difratômetro de raios- $X$. Obteve-se $100 \%$ cobertura por $S P$, as $T^{R} C$ aliviaram com o aumento da temperatura de patamar e o tempo. A maior variação de $T^{R} C$ ocorreu nos primeiros minutos de ensaio, no restante do tempo a $T^{R}$ permaneceu constante. A macrodureza superficial e FWHM das amostras diminuíram com o aumento da temperatura de patamar ao longo do tempo. A macrodureza superficial das amostras e FWHM são maiores após o tratamento de $S P$, antes do aquecimento. O FWHM reduziu ao longo do tempo com mesmo comportamento da queda da $T^{R} C$, correspondente e proporcional à redução da $T^{R} c$. A energia de ativação do processo, calculado pela função Zener-Wert-Avrami não foi coerente com a autodifusão do $F e-\alpha$. Logo, é recomendada a repetição para verificar o resultado. Com base nos dados obtidos, se pode concluir que esta pesquisa possui relevância industrial.

Palavras-chave: Shot-peening; Tensões residuais; DRX fast in-situ; SAE 4140.

\section{THERMAL RESIDUAL STRESS RELAXATION ANALYSIS OF SAE 4140 STEEL BY FAST IN-SITU X-RAY DIFFRACTION}

\section{Abstract}

Compressive residual stresses $\left(T^{R} c\right)$ induced by shot-peening $(S P)$ can be relaxed and redistributed under mechanical, thermal and thermomechanical cyclic loads. This research's aim is to to evaluate the $T^{R}$ creduction by $S P$ through fast in-situ $X$-ray diffraction $\left(D^{R X}{ }_{i n-s i t u}\right)$ on the surface of SAE 4140 steel until the holding time $(t)$ of $t[s]=54000$ in three temperatures $(T): T[K]=423,16 ; 523,16$ e 623,16 . It was investigated the $T^{R} C$ relaxation via thermal loadings, maintaining the samples, previously treated from $S P$, inside a furnace with the different $\mathrm{T}$, while were measured the $T^{R}$ values over the $t$. It was built a vacum chamber to couple in a $X$-ray diffractometer. Results showed $100 \%$ covering by $S P$, the $T^{R}{ }_{c}$ relaxed with the holding temperature and time increasing. The $T^{R}{ }_{C}$ most variation was found in the first minutes test, at the remainder time the $T^{R}$ remained constant. Samples surface macrohardness and $F W H M$ decreased with the holding $T$ over time and are larger after the $S P$ without heating. FWHM decreased over time with the same $T^{R}{ }_{C}$ descreasing, proportional and corresponding to the $T^{R} C$ reduction. Activation energy of the process was computed using Zener-Wert-Avrami function didn't result in the Fe- $\alpha$ self-diffusion energy. So, is necessary new avaliation. Based on the obtained data, it can be concluded that this research has industrial relevance.

Keywords: Shot-peening; Residual stresses; DRX fast in-situ; SAE 4140.

Engenheira de Materiais, Mestranda, LAMEF, PPGE3M - Universidade Federal do Rio Grande do Sul (UFRGS), Porto Alegre - RS, Brasil.

2 Engenheiro Mecânico, Doutorando, UFRGS, Porto Alegre - RS, Brasil.

3 Engenheiro de Materiais, Professor PhD, UFRGS, Porto Alegre - RS, Brasil. 


\section{INTRODUÇÃO}

O processo de tratamento superficial de jateamento conhecido por shot-peening $(S P)$, consiste no disparo de esferas (granalha) de material e de diâmetro prédeterminados na superfície a ser tratada. Como consequência, causam deformações plásticas locais (encruamento) as quais introduzem tensões residuais compressivas $\left(T_{R_{C}}\right)$ na região próxima à superfície do material tratado. Devido à condição de equilíbrio mecânico, essas $T_{R_{C}}$ são balanceadas pela tensão residual trativa $\left(T_{R_{T}}\right)$ em todo o volume do material [1].

Os efeitos benéficos do $S P$ incluem o incremento da vida em fadiga (retardando a nucleação de trincas de fadiga) [1,2] e o aumento da resistência à corrosão [2]. Em contraponto, há o efeito deletério das $T_{B_{C}}$ relaxarem em serviço, na presença de certos elementos cruciais e cotidianos, tais como: alta temperatura $(T)$, cargas mecânicas (cíclicas ou não) / térmicas ou termomecânicas [3]. Além disso, a rugosidade produzida por $S P$ pode danificar a superfície, gerando concentradores de tensões [4].

A rugosidade superficial por $S P$ aumenta com o aumento da intensidade dos tiros. A $T^{R} C /$ Máx é localizada na camada subsuperficial e relaxação térmica das $T^{R} C$ é considerado um tipo de processo de recobrimento térmico [5].

Com o objetivo de estudar o comportamento das $T_{F_{C}}$ como função da temperatura e do tempo de ensaio, foi desenvolvida a técnica de ensaio fast in-situ.

Para alcançar uma alta resolução no tempo (t) durante a análise por difração de raios- $X\left(D^{R X}\right)$, o intervalo de tempo na obtenção dos dados deve ser pequeno 0 suficiente comparado às taxas de mudanças dos processos (a cinética do processo), caracterizando ensaios fast in-situ [6].

Entretanto, as coleções de dados fast in-situ requerem especial instrumentação para uso em elevadas $T$ [6-9]. Por essa razão, foi desenvolvida uma câmara a vácuo acoplada a um difratômetro de raios- $X$, para ensaios fast in-situ $\left(D^{R X} x_{i n-s i t u}\right)$.

Esta pesquisa possui caráter de relevância industrial, uma vez que materiais metálicos podem sofrer aquecimento brusco de acordo com a aplicação em serviço envolvendo aumento de $T$. Gera-se prejuízos tanto à empresa quanto ao consumidor e, muitas vezes, perda do produto. Torna-se essencial o estudo das $T^{R}$ geradas no tratamento de shot-peening e relaxadas pela $T$, visto que a vida útil do produto pode ser aumentada.

O objetivo desta pesquisa é avaliar a redução da $T^{R} c$ obtida por $S P$ na superfície do aço SAE 4140 durante $t[s]=54000$ de patamar em três diferentes $T[K]=423,16$; 523,16 e 623,16 . Entre os objetivos específicos estão: construção de uma câmara de vácuo com aquecimento da amostra em vácuo; desenvolvimento de uma metodologia para ensaios fast in-situ.

\section{Revisão da literatura}

As $T^{R}$, em um dado componente, são tensões elásticas que estão presentes mesmo sem a aplicação de uma carga externa. Tais tensões podem ser devidas às deformações plásticas não-uniformes, gradientes de $T$, gradiente de composição química e diferença de expansão térmica entre as diferentes fases presentes no material, entre outros fatores [10]. As $T^{R}$ possuem mais de um tipo de classificação, conforme a área de abrangência, tais como: $T^{R}$ macroscópicas ( $T^{R}$ Macro), as quais localizam-se em grandes porções volumétricas, quando comparadas com o tamanho de grão do material; $T^{R}$ microscópicas ( $T^{R}$ Micro), cuja distribuição ocorre ao longo de 
um grão e $T^{R}$ submicroscópicas ( $T^{R}$ submicro) que são distribuídas em distâncias interatômicas em uma pequena porção do grão [11]. Em outra classificação: $T^{R}$ Macro ou de primeira ordem ( $T^{R}$ Tipo $\left.I\right)$ e $T^{R}$ intergranulares $\left(T^{R}{ }_{I n t e r g}\right)$ ou de segunda ordem ( $T^{R}$ Tipo II) [11].

Outra definição aborda que o relaxamento da $T_{R_{C}}$ consiste em expor a estrutura $S P$ na $T$ de recobrimento ( $T$ abaixo da metade da $T$ de fusão ( $T^{\text {Fusão }}$ )) sem nenhuma carga mecânica aplicada [12]. Observa-se que a mudança mais significativa na distribuição das $T^{R}$ ocorre na região superficial da estrutura tratada [12].

Medições in-situ já foram empregadas com sucesso por diversos pesquisadores para investigar propriedades de materiais [13], tais como transformações de fases [14], análise de fases [15] e $T_{R}$ [16], através de estudos analíticos, experimentais e computacionais [1-18, 22-25].

Uma pesquisa cuja análise foi relacionada à relaxação das $T^{R} C$ induzidas por $S P$ e a redistribuição, durante ensaios em regime de flexão rotativa de alto e de baixo ciclos em aço carbono $(0,45 \% \mathrm{C})$. As medidas das $T^{R}$ superficiais $\left(T^{R}\right.$ sup $)$ foram efetuadas por difração raios- $X\left(D^{R X}\right)$ antes e durante os ensaios de fadiga. Estas últimas foram obtidas parando o ensaio em ciclos pré-definidos para a amostra ser inserida no equipamento difratômetro de raios- $X\left(D^{R X}\right)$, o qual caracteriza ensaio de $D^{R X}$ ex-situ

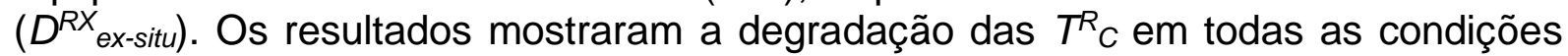
de carga, em função das taxas de relaxamento das $T^{R}$ de acordo com a deformação aplicada [1]. O comportamento de relaxação térmica de metais submetidos ao $S P$ já foi estudado por diversos autores. Feng et al pesquisaram a liga de Titânio (TC4-DT) nas temperaturas de $T[K]=423,26$ e 573,16 em diferentes tempos de envelhecimento $\left(t^{\text {Envelh }}\right)$ [5]. O processo de relaxação foi analisado por $D^{R X}$, bem como através da função Zener-Wert-Avrami. O campo de $T^{R}{ }_{C}$ resultante incluiu quatro parâmetros característicos: tensões residuais superficiais $\left(T^{R} C /\right.$ sup $), T^{R} C /$ Máx, máxima $T^{R}$ de tração $\left(T^{R} T / M a ́ x\right)$, profundidade da $T^{R} C /$ áx e profundidade do campo de $T^{R} C$ [5]. Concluiu-se que a $T^{R} C /$ Máx está localizada na região subsuperficial. Após o envelhecimento, as $T^{R} C$ relaxaram parcialmente, sendo o maior relaxamento na maior $\mathrm{T}$ de ensaio. A máxima taxa de relaxação ocorreu no estágio inicial de aquecimento [5].

Medições fast in-situ das mudanças da composição de fases, tensão e $T^{R}$ foram estudadas ao longo de ciclos de tratamento de aquecimento do aço AISI E52100, incluindo têmpera aplicada com gás $N_{2}[5]$. A $T^{\text {Máx }}[\mathrm{K}]=1203,16$ foi obtida no forno projetado para medições in-situ com alta taxa de aquisição de dados, sendo ao menos um valor a cada $t[s]=3$. Os resultados foram estudados em função da $T$ e do $t$ com desvios explicados pela descarbonetação superficial [5].

Outro estudo avaliou por $D^{R X}{ }_{\text {ex-situ }}$ a estabilidade térmica da $T^{R} C$ e o estado de encruamento na região superficial e subsuperficial no $W C$ cementado ligado ao Cobalto (WC10\%Co) [2]. O material foi tratado por $S P$ nas faixas de $T[K]=823,16$ a 1123,16 por diferentes tempos, sendo o máximo de $t$ máx. [s] $=8400$. 0 comportamento de relaxação da $T^{R}$ e as variações estruturais foram analisadas por método $\operatorname{sen}^{2} \psi$ e por microdureza. Os resultados mostraram que a $T^{R}$, a largura a meia altura (FWHM - full width at half maximum) e a microdureza reduziram drasticamente no estágio inicial de aquecimento e depois diminuíram gradualmente até um estágio estável [3]. A entalpia de ativação da relaxação encontrada para o $W C 10 \%$ Co foi levemente menor que a energia de difusão do $W$ atômico na fase Co $\Delta \mathrm{H}[\mathrm{eV}]=2,5$, enquanto o valor da difusão do Co foi levemente maior que a energia de ativação da auto-difusão do Co de $\Delta \mathrm{H}[e V]=2,7$ [3].

Outra investigação avaliou o tratamento de $S P$ triplo (SPtriplo), utilizando uma menor 
intensidade dos tiros a partir do segundo $S P$, pois pode tanto endurecer quanto suavizar a superfície após o segundo $S P$ [17]. A relaxação foi obtida nas $T$ de aquecimento de $T[K]=573,16 ; 623,16 ; 673,16 ; 723,16$. Além das análises de $T^{R} C$ por $D^{R X}$ antes e depois do SPtriplo, foi efetuada a análise de fase das amostras antes e após o tratamento. Verificou-se a presença de austenita $(A)$ e martensita $(M)$ antes do $S P^{\text {triplo }}$, mas só $M$ depois do $S P^{\text {triplo. }}$ Logo, a austenita retida se transformou em martensita e concluiu-se que é possível a redução da austenita retida próxima a superfície, melhorando as propriedades mecânicas [17].

Outras aplicações de câmaras a vácuo para medições por $D^{R x_{i n-s i t u}}$ incluem o estudo de diferentes parâmetros de nitretação a plasma; geração de fase e crescimento de camadas nitretadas durante ciclos de aquecimento [18]. Pesquisou-se na faixa de $T$ $[K]=723,16$ a 833,16 com duração total do processo de nitretação de $t[s]=10800$ utilizou-se os gases $\mathrm{N}_{2}$ e $\mathrm{H}_{2}$ [18].

\section{MATERIAIS E MÉTODOS}

Esta pesquisa foi realizada nas seguintes etapas: 1) projeto da câmara de vácuo com aquecimento de amostra; 2) produção do aparato; 3) preparação das amostras; 4) ensaios; 5) análises. Na Figura 1 é apresentada a câmara de vácuo aberta e presa no porta-amostras do $D^{R X}$.

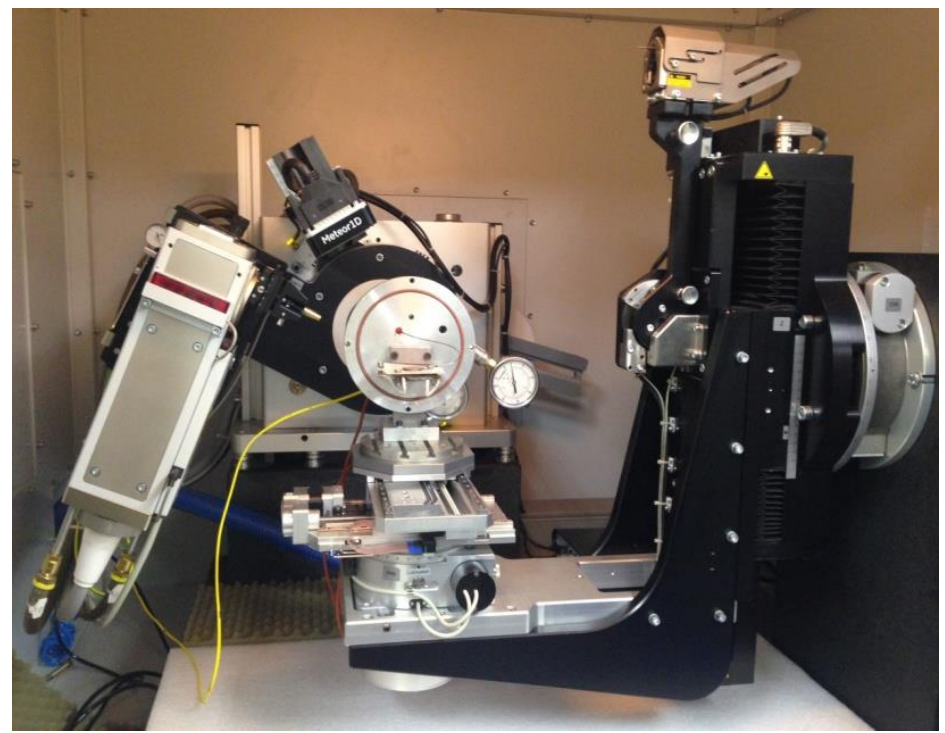

Figura 1. Câmara de vácuo aberta acoplada no difratômetro de raios- $X$ (detector, tubo de raios- $X$, mesa de apoio e goniômetro).

Antes de ser posta em serviço, foram feitas etapas de adaptação da câmara e de verificação dos parâmetros. Primeiro a produção da câmara de vácuo e testes preliminares. Após, o acoplamento e ajustes de parâmetros, para enfim iniciar os ensaios de validação.

Foram efetuados testes antes de acoplar a câmara de vácuo no equipamento de $D^{R X}$ : aquecimento da resistência $\mathrm{NiCr}$ com termopar $K$ (fonte CC-30V/30A); aquecimento da amostra ex-situ com termopar $K$ e fonte $C C$; teste de resistência da película de filme de poliimida Kapton ao aumento de $T$; estanqueidade em pressão positiva e negativa; fuga de corrente; manta isolante térmica de fibra de Aramida.

\subsection{Descrição da câmara de vácuo para altas temperaturas}


O projeto foi realizado no pacote SOLIDWORKS 2016 x64 Edition. Os componentes principais e a instrumentação da câmara de vácuo são: carcaça em alumínio, abertura da janela coberta com filme de poliimida 3M Kapton $($ th $[\mu \mathrm{m}]=30)$, vedação da janela com duas camadas de chapa de aço SAE 1020 com cola de Pulvitec Polystic para altas $T$, resistência de $N i C r$, gás $N_{2}$ (para conferir pureza; atmosfera isenta de gás $\mathrm{O}_{2}$ ), bomba de vácuo Alcatel Pascal $2021 /$ mecânica de duplo estágio, fonte de corrente contínua 30V/30A JBM MP 3030D, passadores: Al para a resistência, nylon para o termopar, de aço inox ISO-KF 25 com 3 entradas (bomba de vácuo, manômetro e vacuômetro), entrada de gás por uma válvula vedada com câmara pneumática, software Analyze de $D^{R X}$, termopar K (Chromel-Alumel com diâmetro de $1(\varnothing[\mathrm{m}]=0,001)$, missangas cerâmicas de alumina isolantes térmicas e elétricas. Porta-amostra de mica entre a resistência e a amostra.

\subsection{Preparação das amostras}

O material utilizado neste estudo foi o aço SAE 4140 fornecido no estado normalizado em barras trefiladas, as quais foram torneadas para produzir as amostras.

Materiais metálicos SAE 4140 são amplamente utilizados na indústria agrícola, automobilística, petrolífera. Por exemplo, na fabricação de eixos hidráulicos, pinos, bielas e virabrequins, rolamentos, engrenagens, porcas e parafusos em máquinas e equipamentos, entre outras aplicações.

Desta barra, foram feitas amostras em forma de discos de dimensões $\emptyset[m]=0,025$ e $L[m]=0,005$. Estes possuem um furo cego que permite a introdução de um termopar $(\varnothing[\mathrm{m}]=0,001$ e folga de 0,002 $\mathrm{m})$ atingindo o centro da amostra de forma radial.

As amostras foram temperadas em óleo a partir da $T[K]=1123,16$ (forno laboratorial Sanches, aquecimento através de resistências nas laterais do forno) mantidas por $t[s]=300$ e revenidas a $T[K]=723,16$ por $t[s]=360 \mathrm{com}$ imersão em grafita.

\subsection{Ensaios}

A aplicação do $S P$ foi gentilmente cedida pela empresa Stihl Ferramentas Motorizadas Ltda., localizada em São Leopoldo/RS. A preparação das amostras, caracterização, análises e o experimento in-situ foram realizados no Laboratório de Metalurgia Física (LAMEF/UFRGS) em Porto Alegre/RS.

\subsubsection{Condições de ensaio}

Foram utilizadas 4 amostras ao total, nomeadas da seguinte forma: amostra 1, amostra 16, amostra 17 e amostra 19. A duração de cada ensaio foi de $t[\mathrm{~s}]=54000$. A amostra 1 não foi relaxada, já as outras permaneceram no intervalo de tempo de $t$ $[s]=54000$ nos patamares de temperatura de $T[K]=423,16 ; 523,16$ e 623,16, respectivamente, inseridas dentro da câmara de vácuo inserida no $D^{R X}$.

\subsubsection{Análise química do aço SAE 4140}

A avaliação da composição química do material como recebido foi obtida pela 
análise química por espectroscopia de emissão atômica no vácuo, segundo a norma ASTM E415 [19].

\subsubsection{Macrodureza}

As macrodurezas das amostras foram executadas pelo método de macrodureza Rockwell C (HRC) ASTM E18 [20]. A macrodureza foi realizada na seção longitudinal das amostras após corte metalográfico. Foi aplicada carga de $\mathrm{m}[\mathrm{kg}]=$ 0,3 por tempo de $t[s]=10$.

\subsubsection{Shot-peening e Metalografia}

O equipamento utilizado no processo de SP foi o AGTOS DT 08-05-3.6.3-01-15 (vide Figura 2). A intensidade Almen [ $\mathrm{mmA}$ ] utilizada foi de 0.02165 , empregadas esferas $S-230$ de aço inox (dureza Vickers $[H V]=700$ ) com diâmetro de $\emptyset[m]=$ 0,006 . O tempo de processo foi de $t[s]=90$.
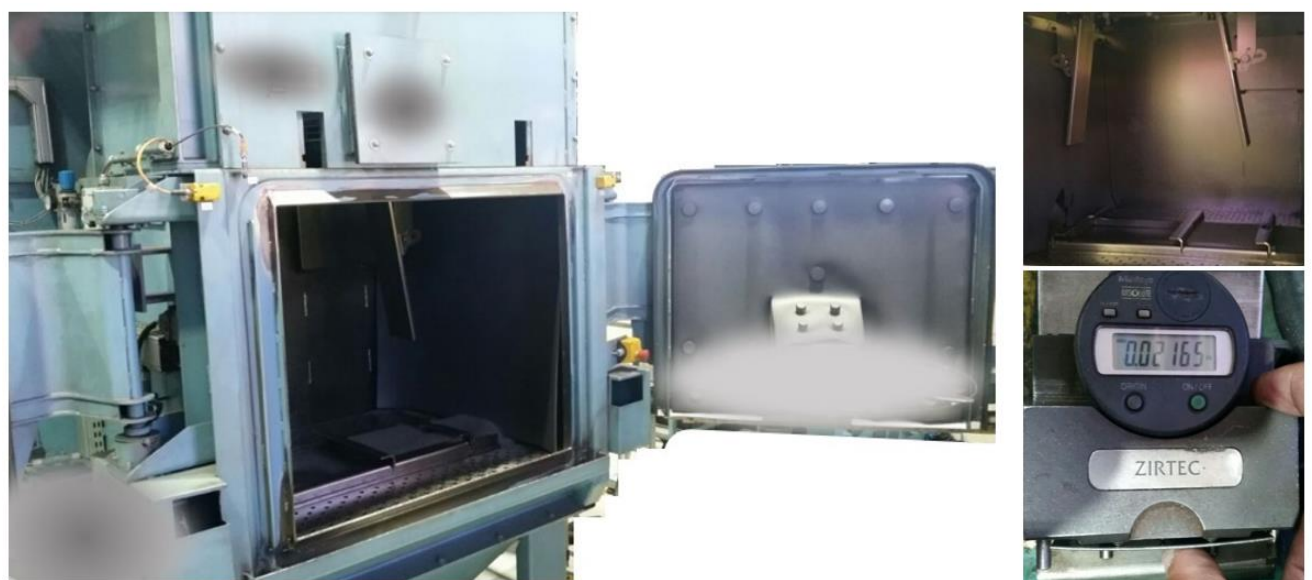

Figura 2. Equipamento de shot-peening modelo AGTOS DT 08-05-3.6.3-01-15. Inclui-se à direita, detalhe do interior da cabine e abaixo à direita a intensidade $A / m e n$ medida no ensaio.

Após o processo de $S P$, foi feita análise metalográfica na seção transversal de uma amostra. A amostra teve a sua seção transversal cortada por disco de corte metalográfico com lubrificação abundante. Após o corte, a seção transversal foi lixada com uma sequência de lixas em ordem crescente de granulometria de \#80 até lixa de número \#1200 e então polida com suspensão de diamante com partículas de tamanho $\varnothing[\mu m]=1$. O ataque químico para revelação da microestrutura foi feito com Nital $2 \%$ alcalino.

\subsubsection{Alívio térmico de tensões residuais e difração de raios- $X$ fast in-situ}

O alívio de tensões foi realizado inserindo as amostras em uma câmara de vácuo acoplada sobre o porta-amostras do equipamento de $D^{R X}$. Os ensaios fast in-situ foram realizados na temperatura de $T[K]=423,16 ; 523,16$ e 623,16 e tempo de patamar de $t[s]=5400$. Esses valores foram estabelecidos de acordo com a revisão da literatura. A pressão positiva do gás $N_{2}$ foi mantida constante e controlada por um manômetro $\left(\mathrm{P}\left[\mathrm{kgf} / \mathrm{cm}^{2}\right]=+1.4\right)$. Esse gás foi usado como purga, alternando com pressão de vácuo na câmara, controlada pelo vacuômetro $(P[m m H g]=-720)$. $O$ termopar $K$ foi conectado no centro do interior da amostra e a aquisição de dados 
obtida via Spider. Para fins de segurança do equipamento de $D^{R X}$, em operação, a $T_{\text {amb }}$ deve estar na faixa de $T[K]=278,16$ a 313,16. A medição do $D^{R X}$ foi adquirida a partir da $T$ de patamar alcançada.

O nível de $T^{R}$ e FWHM foi medido no $D^{R X}$ segundo a norma ASTM E2860 [21], equipamento modelo GE-Seifert-Charon-M research edition com geometria BraggBrentano, utilizando o método $\sin ^{2} \psi$. Devido a câmara de vácuo não permitir a movimentação da amostra, utilizou-se o modo de inclinação ômega, como exemplificado pela Figura 3. O equipamento é composto pelos seguintes dispositivos: goniômetro (em forma circular mede ou constrói os ângulos), colimador (suaviza e direciona os feixes de radiação), câmara focal (define a posição dos eixos para medição de $D^{R X}$ ), porta-amostras (onde foi inserida a câmara de vácuo).

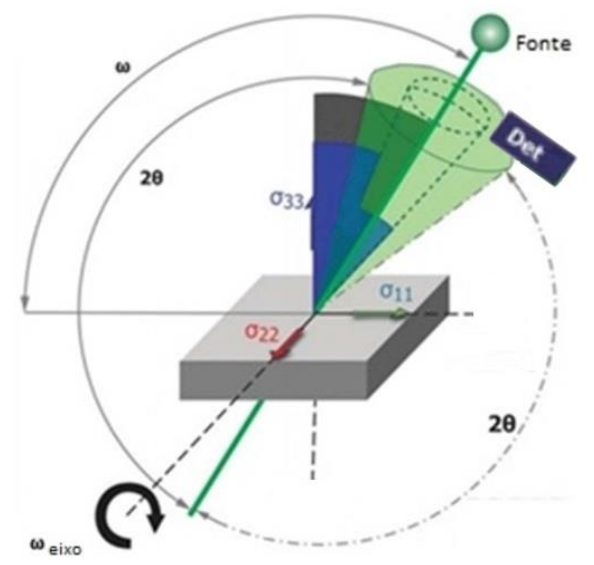

Figura 3. Modo de inclinação ômega. Adaptado de ASTM E2860 [21].

Os parâmetros de medição utilizados foram: radiação $C r-k_{\alpha}$, filtro de vanádio, tensão de $\mathrm{V}[\mathrm{V}]=30000$, corrente I $[A]=0,005$, colimador de $\emptyset[\mathrm{m}]=0,004 \times \mathrm{L}[\mathrm{m}]=0,008$ $\mathrm{m}$, passo de $0,1^{\circ}$, tempo por passo de $\mathrm{t}[s]=4$, cinco inclinações $p s i$ ( $\psi$, do grego) [ $\left.{ }^{\circ}\right]$ $=-45 \mathrm{a}+45$ e a faixa de medição $2 \theta\left[{ }^{\circ}\right]=147$ a 166 . Em cada amostra foi medido um ponto que se situa no centro da respectiva amostra.

A medição foi feita com colimador de $\varnothing[\mathrm{m}]=0,004 \times$ e $L[\mathrm{~m}]=0,008 \mathrm{~m}$. Logo, o diâmetro do $R X$ incidente da amostra foi de $\varnothing[m]=0,004$. Com este colimador obteve-se medições mais rápidas, maiores contagens, por conseguinte melhor determinação do pico e o tempo total de $t[s]=120$ por medição. O software Analyze utilizado para os resultados de $D^{R X}$ faz correções dos fatores geométricos como de polarização e de Lorenz. A determinação da posição dos picos foi feita pelo método do centro de gravidade simples.

Antes de se iniciar as medições in-situ, foi medido o nível de $T^{R}$ de todas as amostras (média e desvio padrão), com o objetivo de ter uma base de comparação para os experimentos in-situ.

\subsubsection{Equação fundamental de tensões com raios- $X$}

A Equação Fundamental [10] para análise de tensões com $R X$, a qual foi utilizada nos diagramas das tensões avaliadas por DRX é mostrada na Equação 1.

onde:

$$
\sigma_{\varphi}=\sigma_{11} \cos ^{2} \varphi+\sigma_{22} \operatorname{sen}^{2} \varphi+2 \sigma_{12} \operatorname{sen} \varphi \cos \varphi
$$

$\varphi=$ define a direção de medida da tensão na superfície da amostra e $\sigma_{i j}=$ 
componentes de tensão.

$\mathrm{Na}$ configuração da Figura 2, somente é medido o $\sigma_{11}$. $\mathrm{O} \sigma_{22}$ não é medido nessa configuração e $0 \sigma_{33}$ não existe, porque a medição é superficial.

\subsubsection{Energia de ativação do alívio térmico por Zener-Wert-Avrami}

A Equação Zener-Wert-Avrami $(Z W A)[5,22]$ mostra a relação do mecanismo termicamente ativado que controla a relaxação das $T^{R}$ Tipo Il, vide Equação 2:

$$
\frac{\sigma_{T, t}^{T R}}{\sigma_{0}^{T R}}=\exp \left[-(A t)^{m}\right]
$$

onde:

$\sigma^{T R} T_{T, t}=T^{R}$ após exposição sob $T, t ; \sigma^{T R} 0=T^{R}$ Inicial da amostra com SP, $m=$ parâmetro numérico dependente do mecanismo de relaxação correspondente; $A=$ função dependente do material e da $T$ de acordo com a Equação 3:

$$
A=B \exp ^{\left(-\frac{\Delta H}{k T}\right)}
$$

onde:

$B=$ constante de velocidade do material; $k=$ constante de Boltzmann; $\Delta H=$ entalpia de ativação para $T^{R}$ real.

De acordo com Equação (2) e os dados experimentais, pode-se determinar $m$ através do coeficiente angular das curvas do gráfico $\log (I n)$ em função de $\log (t)$ [5].

\section{RESULTADOS E DISCUSSÃO}

\subsection{Análise química}

A análise química do material estudado e do padrão são exibidas na Tabela 1.

Tabela 1. Análise química da amostra de SAE 4140 comparada com o padrão SAE.

\begin{tabular}{|c|c|c|c|c|c|c|c|}
\hline Aço & \multicolumn{7}{|c|}{ Elementos químicos (\%) } \\
\cline { 2 - 8 } SAE 4140 & $\mathbf{C}$ & Mn & P & S & Si & Cr & Mo \\
\hline Amostra & $\mathbf{0 . 3 8}$ & $\mathbf{0 . 9 0}$ & $\mathbf{0 . 0 1}$ & $<0.0010$ & $\mathbf{0 . 2 3}$ & $\mathbf{1 . 0 3}$ & $\mathbf{0 . 2 1}$ \\
\hline \multirow{2}{*}{ Padrão } & $\mathbf{0 . 3 8}-$ & $\mathbf{0 . 7 5}-$ & máx. & máx. & $\mathbf{0 . 1 5}-$ & $\mathbf{0 . 8 0}-$ & $\mathbf{0 . 1 5}-$ \\
& $\mathbf{0 . 4 3}$ & $\mathbf{1 . 0 0}$ & $\mathbf{0 . 0 3 0}$ & $\mathbf{0 . 4 0}$ & $\mathbf{0 . 3 5}$ & $\mathbf{1 . 1 0}$ & $\mathbf{0 . 2 5}$ \\
\hline
\end{tabular}

Verifica-se que as amostras desta pesquisa estão dentro das especificações de aços SAE 4140.

\subsection{Metalografia}

A Figura 4 apresenta a superfície obtida de uma amostra representativa após o ensaio fast in-situ no $\mathrm{D}^{\mathrm{RX}}$ na $\mathrm{T}[\mathrm{K}]=423,16$ e no $\mathrm{t}[\mathrm{s}]=54000$. 


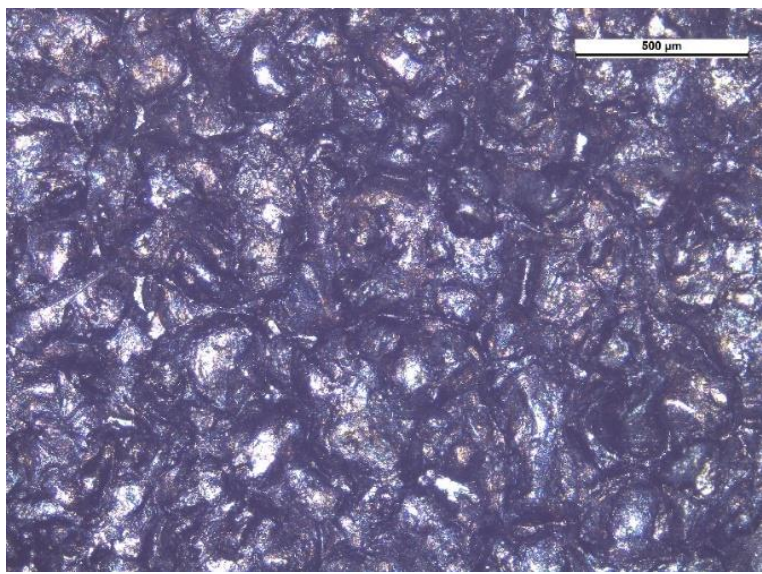

Figura 4. Superfície obtida no microscópio óptico de $100 \%$ de cobertura por SP e sem ataque. Aumento de 5 vezes na imagem original.

É possível visualizar, através da Figura 4, que $100 \%$ da cobertura superficial da amostra foi exposta ao shot-peening [25]. Este resultado era desejado, visto que é ideal o tratamento de SP com $100 \%$ da superfície coberta [24].

\subsection{Dureza}

A Figura 5 mostra a macrodureza Rockwell $C$ de cada amostra após os ensaios de relaxação térmica.

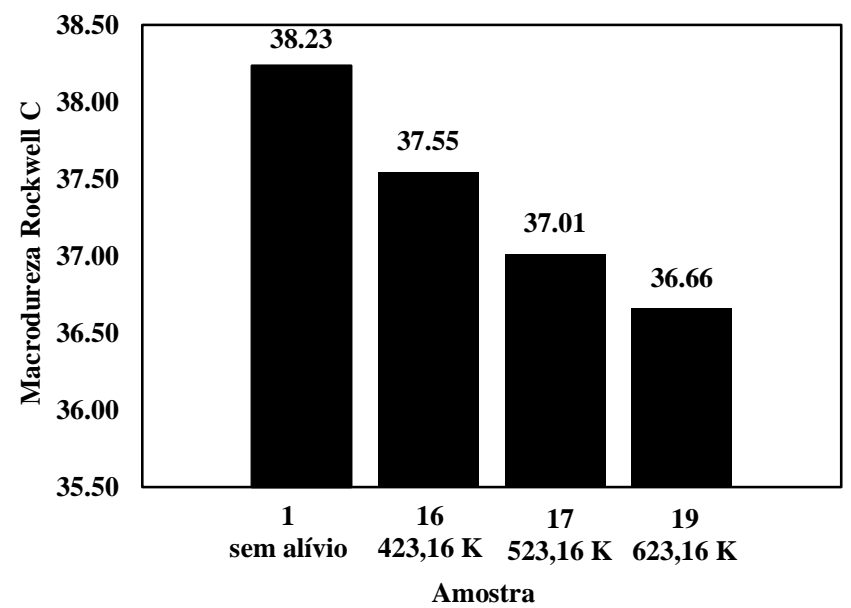

Figura 5. Macrodureza das amostras $(1,16,17,19)$ após os ensaios fast in-situ.

Observa-se na Figura 5 a queda da dureza com a $T$ em relação ao valor inicial para os três patamares de $T$. O resultado das macrodurezas das amostras 16,17 e 19 foram comparados em relação ao valor inicial de macrodureza, representado pela amostra 1, sem alívio térmico. Esta queda foi mais brusca na amostra 19, cuja $T$ de relaxação $(T[K]=623,16)$ foi a maior, intermediária na amostra 17 que foi submetida à $T$ de relaxação mediana de $T[K]=523,16$ e foi menos acentuada na amostra 16 a qual permaneceu na menor $T$ de ensaio $(T[K]=423,16)$. Logo, houve o declínio das macrodurezas inversamente proporcional ao aumento das $T$ de ensaio fast in-situ [3]. Este fenômeno é coerente com as características do ensaio fast in-situ, o qual ocorrem os mecanismos de relaxação das $T^{R} C$ em função da $T$ do ensaio e do $t$. 


\subsection{Alívio térmico de tensões residuais fast in-situ superficial e subsuperficial}

A Figura 6 mostra a variação da $T^{R}$ em função do $t$ para as $T$ de $T[K]=423,16$; 523,16 e 623,16 .

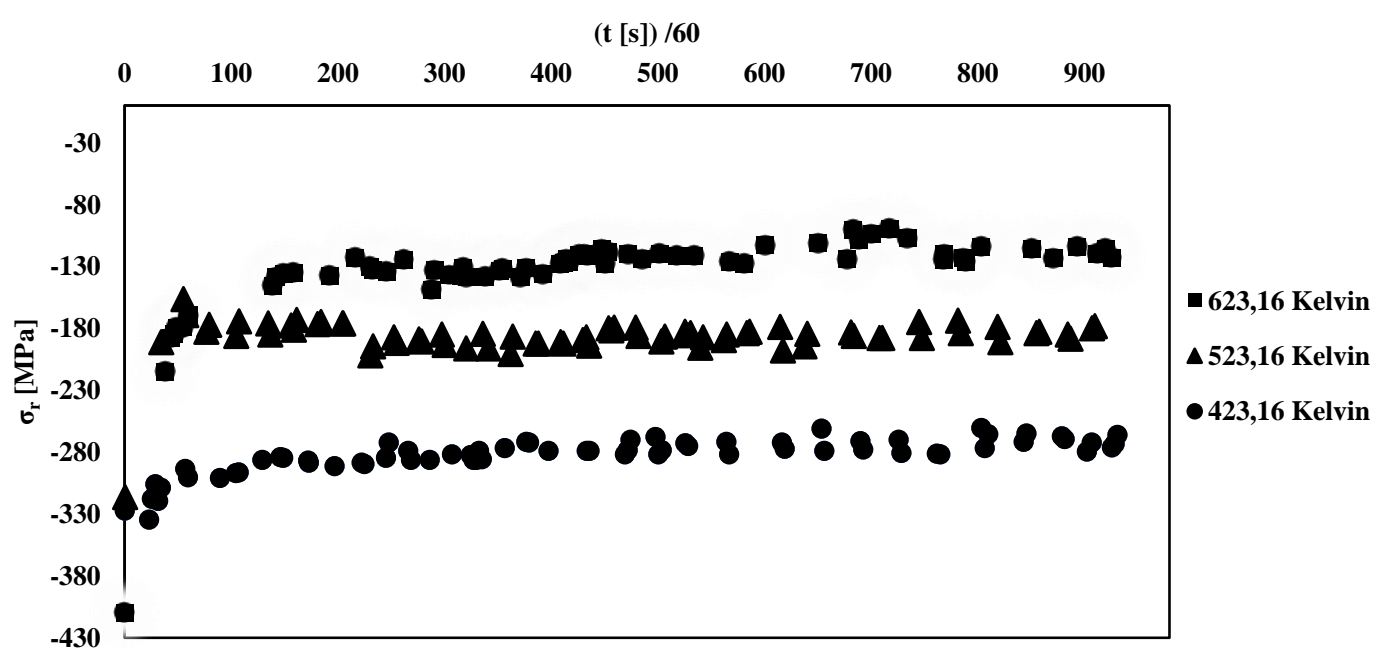

Figura 6. Variação da tensão residual em função do tempo nas temperaturas de $T[K]=423,16$; 523,16 e 623,16 .

O comportamento de queda da $T^{R}$ confirmou o resultado obtido nas microdurezas das amostras. A redução mais acentuada da $T^{R}$ foi da amostra 19 , seguida pelas amostras 17 e 16. Desta forma, as quedas das $T^{R}$ foram inversamente proporcionais ao acréscimo das $T$. A principal queda ocorreu nos primeiros minutos de ensaio até chegar a um patamar de $T^{R}$ constante. A $T^{R}$ Final permaneceu sendo compressiva, apesar da queda de 18,94 \% para amostra 16, 43,73 \% para amostra 17 e 69,95\% para amostra 19 , em relação ao valor inicial da tensão residual compressiva. A $^{\mathrm{R}_{\text {Final }}}$ permaneceu sendo compressiva para as três amostras.

O processo de relaxação térmica é atribuído à redução da $T^{R}$ causando desencaixe plástico (conhecido em inglês como plastic misfit) por difusão ou movimento de discordâncias dos átomos, levando à redução da energia armazenada [5]. Esses mecanismos envolvem aniquilação de defeitos de rede metaestáveis, rearranjo de discordâncias controladas por fluência e recristalização em temperaturas altas [5].

Pesquisadores também propuseram o mecanismo de relaxação como sendo dividido em dois estágios: I) o primeiro estágio, chamado estágio quasi-estático (do inglês: shakedown elastic) devido às redistribuições das deformações plásticas nas subcamadas afetadas, ii) o segundo estágio é a relaxação gradual devido, principalmente, à evolução das propriedades mecânicas sob cargas cíclicas [12].

Em relação às $T^{R}$ observou-se que a energia armazenada no $S P^{\text {triplo diminuiu com o }}$ aumento do $t$ de recobrimento, indicando que a energia armazenada alivia quando as discordâncias geradas no $S P$ recobrem [17]. As relaxações térmicas são causadas por processos termicamente ativados, de tal forma que o alívio da energia armazenada fornece a força motriz para recuperação térmica e a recristalização [17].

\subsubsection{Largura a meia altura (FWHM)}

A Figura 7 apresenta o FWHM versus tempo para as três condições de T. 


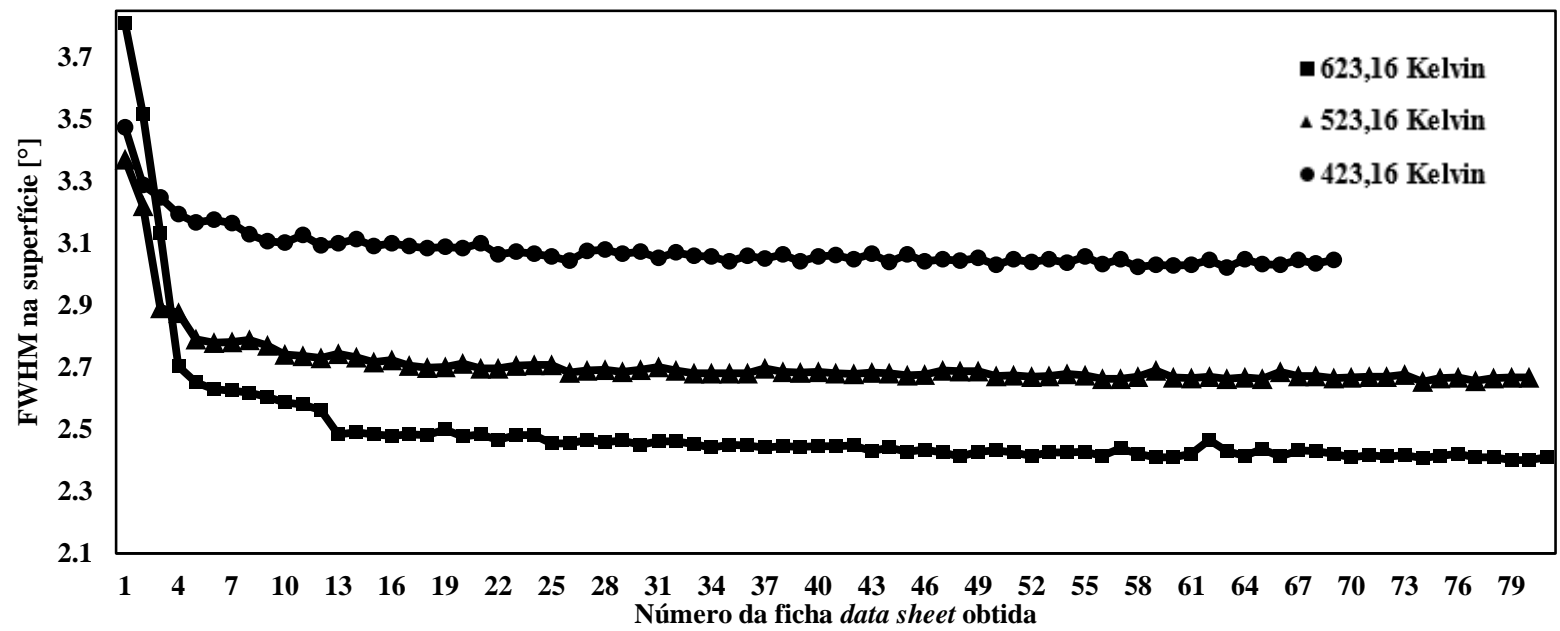

Figura 7. Largura a meia altura $(F W H M)$ em função do $t$ para as amostras expostas às $T[K]=$ $423,16 ; 523,16$ e 623,16$)$.

De acordo com a Figura 7, o FWHM reduziu inversamente proporcional ao aumento da $T$ e do $t$ de ensaio. Este resultado corrobora com os resultados de $T^{R}$ e de macrodurezas. O estudo dos resultados de FWHM é utilizado para caracterizar o efeito do encruamento da camada superficial deformada, particularmente em componentes sujeitos ao $S P$, sendo que o FWHM reduz com o aumento da $T$ e do $t$ [3]. Vale ressaltar que o FWHM está intimamente relacionado a relaxação da $T^{R}$ [3].

\subsection{Energia de ativação do processo de aquecimento}

A partir do gráfico $\log (\ln )$ versus de $\log (t)$ (vide Figura 8) é possível obter 0 coeficiente angular $m$ das curvas de aquecimento para cada $T$ e solucionar a equação $Z W A$ da energia de ativação.

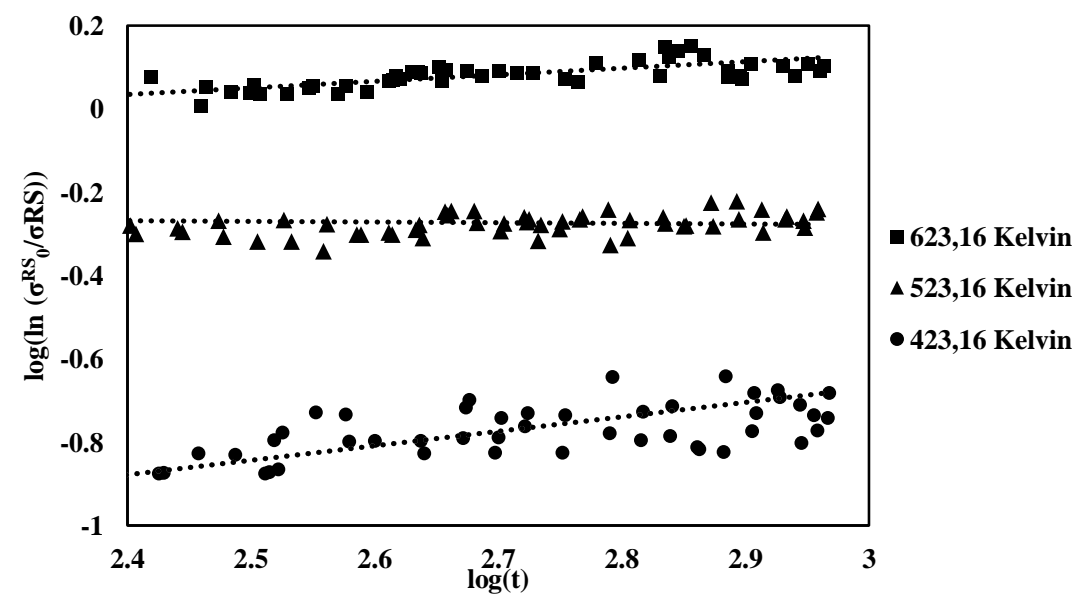

Figura 8. Dados lineares para determinação do coeficiente angular " $m$ " com propósito da resolução da equação Zener-Wert-Avrami.

Como apresentado na Figura 8, os dados medidos de $T^{R}$ estão de acordo com a relação linear [5]. Desta forma, torna-se possível o cálculo do valor dos coeficientes angulares $m$, a partir para as três curvas de aquecimento (Tabela 2).

Tabela 2. Coeficiente angular da curva da aquecimento fast in-situ. 


\begin{tabular}{lccc}
\hline Temperatura [K] & $\mathbf{4 2 3 , 1 6}$ & $\mathbf{5 2 3 , 1 6}$ & $\mathbf{6 2 3 , 1 6}$ \\
\hline$m$ & 0,3475 & $-0,0157$ & 0,1576 \\
[adimensiona] & & & \\
\hline
\end{tabular}

Através do cálculo das Equações 1-3 e fazendo uso dos resultados da Tabela 2, a energia de ativação $\Delta \mathrm{H}[\mathrm{el}]$ obtida em cada $T$ de ensaio é mostrada na Tabela 3.

Tabela 3. Energia de ativação do aquecimento fast in-situ.

\begin{tabular}{lccc}
\hline Temperatura [K] & $\mathbf{4 2 3 , 1 6}$ & $\mathbf{5 2 3 , 1 6}$ & $\mathbf{6 2 3 , 1 6}$ \\
\hline$\Delta H[e /]$ & 3,94 & 3,64 & 1,63 \\
\hline
\end{tabular}

É possível associar este resultado ao mecanismo termicamente ativado que controla a relaxação das $T^{R}$ Tipo II. Desta forma, o resultado obtido deveria indicar o mecanismo da autodifusão do $\mathrm{Fe}-\alpha$, sendo energia de ativação padrão de $\Delta H^{F e-\alpha}[e V]=2,6$, verificado na revisão [22]. Os resultados foram próximos para as três $T$ e superior ao valor esperado. Logo, é necessário verificar qual a fonte de erro. Podendo ser no experimental ou na parte analítica da pesquisa.

\section{CONCLUSÕES}

Com base nas condições de ensaio realizadas nesta pesquisa, concluiu-se que:

- As amostras foram expostas ao SP obtendo $100 \%$ de cobertura.

- A macrodureza superficial das amostras submetidas a shot-peening diminuiu com o aumento do patamar de temperatura e tempo. As amostras 16, 17 e 19 diminuíram da dureza inicial $[H R C]$ de 38,23 para a dureza final $[H R C]$, após $t[s]=54000$ de: 37,$55 ; 37,01$ e 36,66 , respectivamente.

- As tensões residuais compressivas iniciais aliviaram com aumento do patamar de temperatura e tempo. As amostras 16,17 e 19 diminuíram da $\mathrm{T}^{\mathrm{R}}$ Inicial $[\mathrm{MPa}$ ], em $\mathrm{t}[\mathrm{s}]$ $=0$, de: $-327,3 ;-316,3$ e $-410,0$, respectivamente; para o valor final de $T^{R}$ Final $[M P a]$, após $\mathrm{t}[\mathrm{s}]=54000$ de: $-265,9 ;-178,0$ e $-123,2$, respectivamente.

- O FWHM sofreu queda com aumento do patamar de temperatura e de tempo. As amostras 16, 17 e 19 diminuíram de $F W H M^{\text {|nicial }}$ [ $]$, em t [s] = 0, de: 3,47; 3,28 e 3,81, respectivamente; para o valor final de $F W H M^{\text {Final }}\left[{ }^{~}\right]$ de, após $t[s]=54000$ de: 3,04; 2,67 e 2,41, respectivamente.

- O comportamento da relaxação das tensões residuais compressivas e do FWHM tiveram a mesma tendência. A maior variação das tensões residuais e do FWHM ocorreu nos primeiros segundos $t[s]=360$ de ensaio para as três amostras, no restante do tempo a tensão residual e FWHM permaneceram constantes.

- A energia de ativação do processo, de acordo com a revisão, deveria ser da autodifusão do Fe-a. Logo, é necessário repetir o experimental e os cálculos analíticos.

\section{Agradecimentos}

Ao CNPq, à Stihl Ferramentas Motorizadas Ltda. e ao LAMEF pela infraestrutura e apoio.

\section{REFERÊNCIAS}


1 Kim J, Cheong S, Noguchi H. Residual stress relaxation and low- and high-cycle fatigue behavior of shot-peened medium carbon stress. International Journal of Fatigue. 2013; 56:114-122.

2 Gibson G J, Perkins K M, Gray S, Leggett A. J. Influence of shot peening on hightemperature corrosion and corrosion-fatigue of nickel based superalloy $720 \mathrm{Li}$. Materials at High Temperatures. 2016;33:225-233.

3 Wang C, Jiang C, Ji V. Thermal stability of residual stresses and work hardening of shot peened tungsten cemented carbide. Journal of Materials Processing Technology. 2017;240:98-103.

4 Lago J, Guagliano M, Bokůvka O, Trško L, Řidký O. Nový F, Závodská D. Improvement of fatigue endurance of welded S355 J2 structural steel by severe shot peening. Surface Engineering. 2017,0:1-6.

5 Feng BX, Mao XN, Yang GJ, Yu LL, Wu XD. Residual stress field and thermal relaxation behaviour of shot-peened TC4-DT titanium alloy. Materials Science \& Engineering A. 2009;512:105-108.

6 Rocha, AS, Hirsch, T. Fast in situ X-ray diffraction phase and stress analysis during complete heat treatment cycles of steel. Materials Science \& Engineering $A$. 2005;395:197-207.

7 Valkenburg JAV, McMurdie HF. High-Temperature X-ray Diffraction Apparatus. Journal of reasearch (Part of the Journal of Research of the National Bureau of Standards). 1947;38:415-418.

8 Spreadborough J, Phil D, Christian J W. High-temperature X-ray diffractometer. Journal of scientific instruments. 1959;36:116-118.

9 Hirsch T, Clarke TGR; Rocha AS. An in-situ study of plasma nitriding. Surface \& Coatings Technology. 2007;201:6380-6386.

$10 \mathrm{Lu} \mathrm{J}$ ed. Handbook of measurement of residual stresses. Fairmont Press. 1996.

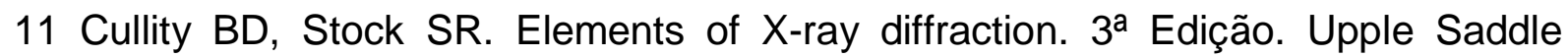
River; Prentice Hall; 2001.

12 Seddik R, Seddik M, Atig A, Fathallah R. Thermo-mechanical relaxation of compressive residual stresses induced by shot peening. Procedia Structural Integrity. 2016;2:2182-2189.

13 Dolan MD, Zdzieszynski S, Misture ST. A High-temperature Powder Diffraction Furnace. International Centre for Diffraction Data. Advances in X-Ray Analysis. 2003;46:50-55.

14 Valkenburg JAV, McMurdie HF. High-Temperature X-ray Diffraction Apparatus. Journal of reasearch (Part of the Journal of Research of the National Bureau of Standards). 1947;38:415-418.

15 Epp J, Surm H, Kessler O, Hirsch T. In situ X-ray phase analysis and computer simulation of carbide dissolution of ball at different austenitizing temperatures. Acta Materialia. 2007;55:5959-5067.

16 Jinxiang L, Huang Y. Prediction of residual stress relaxations in shot peened specimens and its application for the rotor disc assessment. Materials Science \& Engineering A. 2010;527:6690-6698.

$17 \mathrm{Fu} \mathrm{P}$, Jian $\mathrm{C}$. Residual stress relaxation and micro-structural development of the surface layer of $18 \mathrm{CrNiMo7-6}$ steel after shot peening during isothermal annealing. Materials and Design. 2014;56:1034-1038.

18 Hirsch T, Clarke T G R; Rocha A S. An in-situ study of plasma nitriding. Surface \& Coatings Technology. 2007;201:6380-6386.

19 ASTM E415. Standard Test Method for Atomic Emission Vacuum Spectrometric Analysis of Carbon and Low-Alloy Steel", ASTM International. 
20 ASTM E18. Standard Test Methods For Rockwell Hardness Of Metallic Materials, ASTM International.

21 ASTM E2860. Standard Test Method for Residual Stress Measurement by X-Ray Diffraction for Bearing Steels, ASTM International.

22 Menig R, Schulze V, Vöhringer O. Effect of Short-Time Annealing on Fatigue Strength of Shot-Peened AISI 4140 in a Quenched and Tempered Material State. Shot Peening. 2003: 331-337.

23 Totten G E. Handbook of Residual stress and Deformation of Steel. ASM International, Residual stresses. 2002.

24 Tan L, Zhang D, Yao C, Wu d, Zhang J. Evolution and empirical modeling of compressive residual stress profile after milling, polishing, and shot peening for TC17 alloy. Journal of Manufacturing Processes. 2017;26:155-165.

25 Sherafatania K, Farrahi G H, Mahmoudi A H, Ghasemi A. Experimental measurement and analytical determination of shot peening residual stresses considering friction and real unloading behavior. Materials Science \& Engineering A. 2016;657:309-321. 